Vinajak Gopi, Barbara Milles, Eberhard Spanuth, Matthias Müller-Hennessen, Moritz Biener, Kiril Stoyanov, Norbert Frey and Evangelos Giannitsis*

\title{
Comparison of the analytical performance of the PATHFAST high sensitivity cardiac troponin I using fresh whole blood vs. fresh plasma samples
}

https://doi.org/10.1515/cclm-2021-0354

Received March 24, 2021; accepted May 25, 2021;

published online June 7, 2021

\section{Abstract}

Objectives: The PATHFAST hs-cTnI (high-sensitivity cardiac troponin) assay is the first point-of-care assay with a high-sensitivity designation that received FDA approval for diagnosis of myocardial infarction (MI). Testing from whole blood does not need centrifugation and therefore is faster and more convenient in the emergency room instead of plasma. However, there is sparse evidence whether point-of-care testing of Tn from whole blood is as reliable as from plasma samples.

Methods: We investigated the agreement between plasma and whole blood hs-cTnI by using the PATHFAST hs-cTnI assay. Hs-cTnT measured on Cobas 602 in the central laboratory and compared to a final diagnosis of NSTEMI using serial hs-cTnT served as reference. We assessed biases, limits of agreement $( \pm 1.96 \mathrm{SD})$ and coefficients of correlation, and tested the discriminatory ability of the baseline sample of plasma and whole blood hs-cTnI and plasma hscTnT to discriminate non-ST-segment elevation myocardial infarction (NSTEMI).

Results: A total of 224 paired fresh samples were collected simultaneously from 191 patients presenting with suspected acute coronary syndrome. There was an excellent correlation between plasma and whole blood hs-cTnI ( $r=0.99)$, and a very good inter-rater agreement $(\mathrm{k}=0.93)$ between elevated

Vinajak Gopi and Barbara Milles contributed equally to this work.

*Corresponding author: Prof. Evangelos Giannitsis, Department of Cardiology, Angiology and Pulmonology, Heidelberg University Hospital, Im Neuenheimer Feld 410, 69120 Heidelberg, Germany, Phone: +49 6221 568670, Fax: +496221 565516,

E-mail: evangelos.giannitsis@med.uni-heidelberg.de Vinajak Gopi, Barbara Milles, Matthias Müller-Hennessen, Moritz Biener, Kiril Stoyanov and Norbert Frey, Department of Cardiology, Angiology and Pulmonology, Heidelberg University Hospital, Heidelberg, Germany

Eberhard Spanuth, DIAneering GmbH, Heidelberg, Germany and normal plasma and whole blood results. Precision evaluation according to CLSI ep 15 revealed comparable coefficients of variation (CV) in whole blood and plasma. The discriminatory ability of baseline hs-cTnT, plasma and whole blood hs-cTnI was excellent (AUC 0.967, AUC 0.954 and AUC 0.953) without significant difference.

Conclusions: Whole blood can be used interchangeably with plasma for more convenient and less time and laborconsuming testing of hs-cTnI on the PATHFAST instrument.

Keywords: high-sensitivity; method comparison; plasma; point-of-care; troponin; whole blood.

\section{Introduction}

Point-of-care (POC) cardiac troponin (cTn) assays are recommended when the central laboratory is unable to provide laboratory results timely to the emergency department (ED), or when central laboratory cTn measurements are temporarily or permanently not available [1]. The high number of patients admitted with unspecific chest pain or dyspnea of unknown etiology prompts the need to shorten the time to diagnosis, and the length of stay in order to decongest overcrowded EDs [2].

However, until recently POC cTn assays did not meet the International Federation of Clinical Chemistry and Laboratory Medicine Task Force on Clinical Applications of BioMarkers (IFCC TF-CB) criteria required for a high sensitivity designation [3], and therefore POC cTn assays were only cleared by the Federal Drug Administration (FDA) as an aid to rule-in an MI but were unable to safely rule-out an MI due to inappropriately low analytical sensitivities. Among the increasing number of POC tests that achieve a comparable analytical sensitivity and precision like central laboratory instruments [4-7], the PATHFAST hs-cTnI (high-sensitivity cardiac troponin) assay (LSI Mediance, Japan) was the first instrument and assay that was cleared by the FDA $[8,9]$.

Using validated thresholds and concentration changes, PATHFAST hs-cTnI allows a similar diagnostic performance for rule-in and rule-out of MI using the 
ESC 0/1 h protocol like the Abbott Architect hs-cTnI assay [5]. However, until now, the clinical performance of PATHFAST hs-cTnI was evaluated under optimal experimental conditions by testing batch-wise from frozen plasma aliquots, and not from fresh whole blood specimen.

According to experience, the main application in practice in the emergency department is POC testing from whole blood. Therefore, the aim of the present study was to compare hs-cTnI concentrations measured immediately from fresh plasma and whole blood directly, and to evaluate the clinical performance compared to an adjudicated diagnosis based on the Roche hs-cTnT central laboratory assay.

\section{Materials and methods}

We enrolled 191 patients with suspected ACS presenting to the ED of the University hospital of Heidelberg between January 28th, 2020 and August 28th, 2020. All samples were collected prospectively and simultaneously. Patients with ST segment elevation MI (STEMI) were not excluded for comparison of plasma and whole blood concentrations but were excluded for assessment of clinical performance. Besides STEMI, only patients with end-stage renal disease on hemodialysis were excluded.

\section{Gold standard diagnosis}

A standard electrocardiogram was collected at admission and was interpreted by trained medical staff. cTn was routinely measured on admission and after $1 \mathrm{~h}$, and at later time-points whenever the diagnosis was equivocal based on the $0 / 1 \mathrm{~h}$ results. Measurements were performed in the central laboratory of the University hospital using the Roche hs-cTnT assay using heparin plasma, measured on a Cobas 602 instrument (Elecsys ${ }^{\circledR}$ troponin T high-sensitive, Roche Diagnostics). A study-specific blood draw for the additional measurement of hs-cTnI was routinely collected at baseline at the same time point with the hscTnT measurement, and sporadically after $1 \mathrm{~h}$. The final diagnosis was based on the serial hs-cTnT results and on all available clinical and imaging results, invasive coronary angiogram, electrocardiogram and routine laboratory testing. In particular, the diagnosis of a non-ST segment myocardial infarction (NSTEMI) was made according to the

Table 1: Inter-rater agreement between elevated hs-cTnl in plasma and whole blood.

\begin{tabular}{lrrr}
\hline Elev hs-cTnl whole blood & \multicolumn{2}{c}{$\begin{array}{c}\text { Elev hs-cTnl plasma } \\
\text { >24.2 } \mathbf{~ n g / L ~}\end{array}$} & Total number \\
\cline { 2 - 3 } & No & Yes & \\
\cline { 2 - 3 } & 133 & 0 & 133 \\
\hline No & 7 & 84 & 91 \\
Yes & 140 & 84 & 224 \\
\hline
\end{tabular}

The comparison yields a Cohen's Kappa of kappa $=0.93(95 \% \mathrm{Cl}$ : $0.89-0.98)$ which indicates a very good agreement $(>0.90)$ between methods. fourth definition of myocardial infarction [10]. The final diagnosis of all patient conditions was adjudicated by two trained cardiologists (MM, MB) independently. In cases of disagreement, a third cardiologist (EG) reviewed the case.

\section{POC device, principle of analysis and analytical performance}

All measurements hs-cTn assays were performed from fresh biomaterial during routine measurements at the central laboratory of the University of Heidelberg for hs-cTnT, and in the research laboratory for cardiac biomarkers in the Department of Cardiology for hs-cTnI. The limit of detection (LoD) of hs-cTnT on the Cobas 602 is $3.0 \mathrm{ng} / \mathrm{L}$, and the 99th percentile has been described at $14 \mathrm{ng} / \mathrm{L}$ in a multicentric reference study [11]. The lowest concentration with a CV less than or equal to $10 \%$ (Limit of Quantitation) with the Roche hs-cTnT assay was $13 \mathrm{ng} / \mathrm{L}$ [12]. In an appropriately sized reference study of 626-740 healthy individuals, the rate of detectable hs-cTnT was $81.4-83.3 \%$ and $96.5-96.9 \%$ in females and males, respectively [13].

The PATHFAST hs-cTnI utilizes a chemiluminescent enzyme immunoassay (CLEIA) and a magnetic particle separation method that handles target substances only (MAGTRATION ${ }^{\circledR}$ technology) to quantitatively measure the cTnI concentration in Li-heparin, $\mathrm{Na}$-heparin, or EDTA- $\mathrm{K}_{2}$ whole blood or plasma. Alkaline phosphatase labeled monoclonal antibody and anti-cTnI monoclonal antibody coated magnetic particles are mixed with the sample. cTnI containing specimen binds to anti-cTnI antbodies forming immune-complexes with enzyme labeled antibodies and antibodies coated with magnetic particles. After removing the unbound enzyme labeled antibodies from the reaction mixture a chemiluminescent substrate is added. After incubation the luminescence by the enzyme reaction is detected. The intensity of measured luminescence is proportional to the cTnI concentration of the sample. The limit of blank (LoB) was determined in accordance with CLSI guideline EP 17 by measuring 60 blank samples and calculating the mean and standard deviation (SD) according to the following: $\mathrm{LoB}=$ mean $+1.645 \times \mathrm{SD}$.

The mean was $-0.916 \mathrm{ng} / \mathrm{L}$ and SD was $1.30 . \mathrm{LoB}=-0.916+$ $(1.645 \times 1.30)=1.223 \mathrm{ng} / \mathrm{L}$.

For LoD calculation, the pooled SD was $0.673 \mathrm{ng} / \mathrm{L}$ and with the equation $\mathrm{LoD}=\mathrm{LoB}+(1.645 \times \mathrm{SD}) \mathrm{LoD}=1.223+(1.645 \times 0.673)=2.33 \mathrm{ng} / \mathrm{L}$. According to the manufacturer, the test-specific LoD measured in plasma is $2.33 \mathrm{ng} / \mathrm{L}$, the sex-independent overall 99th percentile are $28 \mathrm{ng} / \mathrm{L}$ (90\% CI, 20-30) (20 ng/L (90\% CI, 13-30) in 352 women and $30 \mathrm{ng} / \mathrm{L}$ (90\% CI, 21-37) in $382 \mathrm{men}$ ) with a coefficient of variation of $10 \%$ or less at $11.0 \mathrm{ng} / \mathrm{L}$. The measurable number of healthy subjects between LoD and 99th percentile was 487 (66.3\%) from 734 healthy subjects [9]. Another cohort of more than 300 males and 300 females yielded a sexindependent 99th percentile of $24.2 \mathrm{ng} / \mathrm{L}$ (95\% CI, 17.6-27.4) overall, $21.1 \mathrm{ng} / \mathrm{L}$ (95\% CI, 13.4-25.3) in females and $27.0 \mathrm{ng} / \mathrm{L}$ (95\% CI, 18.5-27.7) in men. The limit of quantitation (LoQ) at $20 \% \mathrm{CV}$ is $4 \mathrm{ng} / \mathrm{L}$ and at $10 \%$ CV $15 \mathrm{ng} / \mathrm{L}$ [5]. All results were obtained from plasma samples.

\section{Method comparison of hs-cTnl between whole blood and plasma}

Concentrations of hs-cTnI were measured with the PATHFAST hs-cTnI assay. The EDTA whole blood samples were drawn at presentation in 
all patients. The collection tubes containing whole blood were sent immediately to the research laboratory.

After mixing gently the collection tube, $0.1 \mathrm{~mL}$ of whole blood was separated into a sample well of a cartridge. After loading the cartridge, the PATHFAST hs-cTnI assay was started. If necessary, six cartridges could be measured in parallel. During the measurement of the whole blood sample (duration approx. $17 \mathrm{~min}$ ) the remaining whole blood was processed into plasma by $10 \mathrm{~min}$ centrifugation at 2,000 g. After separation of the plasma, $0.1 \mathrm{~mL}$ were dispensed into a sample well of a cartridge. After loading the cartridge, the measurement was started. This procedure ensured the smallest possible time difference between whole blood and plasma measurement.

Besides these procedure the precision of the PATHFAST hs-cTn assay was determined in parallel from whole blood and plasma at different concentrations and on different days.

\section{Clinical performance of plasma and whole blood compared to the reference based on serial hs-cTnT}

The results of the POC assay were compared with the Roche hs-cTnT assay on a Cobas 602 which served as the reference laboratorybased method. All measurements hs-cTn assays were performed from fresh biomaterial during routine measurements at the central laboratory of the University of Heidelberg for hs-cTnT. The study protocol was approved by the local Ethical Committee of the University of Heidelberg, and all patients provided written informed consent.

\section{Statistical methods}

Continuous variables were tested for normal distribution using the Kolmogorov-Smirnov test, and were presented either as means with standard deviations or $95 \%$ confidence intervals (CI), or as medians with interquartile range.

Method comparison between plasma and whole blood was calculated using the non-parametric Passing-Bablok regression [14]. Results are displayed as a scatter diagram and regression line. The slope B and intercept A were calculated with their 95\% confidence interval. A Bland-Altman plot was used to compare plasma and whole blood measurements in order to detect the presence of a systematic bias, and to identify possible outliers across a representative concentration range. The limits of agreement (LoA) were defined as the mean difference plus and minus 1.96 times the standard deviation of the differences.

The areas under the receiver-operating characteristic curves were constructed for discrimination of the final diagnosis of NSTEMI and compared for baseline plasma and whole blood hs-cTnI and baseline hs-cTnT on the central laboratory analyser as recommended by DeLong et al. The inter-rater agreement between plasma and whole blood samples above and below the 99th percentile values were calculated using statistic Kappa ( $\kappa$ ) and weighted Kappa according to Cohen.

All analyses were performed with Medcalc 17.6 (MedCalc Software, Ostend, Belgium).

\section{Results}

\section{Patients characteristics}

We collected 318 paired samples from 191 patients. The mean age was $63.7 \pm 16.6$ years, and 105 participants (55.9\%) were male. Among the 318 collected paired samples, 94 had to be excluded due to missing values or concentrations below the LoD for either plasma or whole blood hs-cTnI or for hs-cTnT.

The final diagnosis was NSTEMI in 52 patients (27.7\%), unstable angina in 15 (8.0\%), and non-cardiac chest pain in 59 cases $(31.4 \%)$. Patients with NSTEMI were significantly older (68 years \pm 16.5 vs. 62.0 years $\pm 16.4 ; p=0.02$ ), with significantly more males than females $(n=36(69.2 \%)$ vs. $\mathrm{n}=16$ (30.8\%), $\mathrm{p}=0.023)$. Rule-in diagnoses other than NSTEMI included pulmonary embolism, myocarditis, Tako Tsubo Cardiomyopathy.

\section{Method comparison between plasma and whole blood}

Of 318 paired samples, 94 samples were not eligible due to missing information on hs-cTnI, or hs-cTnI values below the LoD of $2.33 \mathrm{ng} / \mathrm{L}$, thus disqualifying the complete sample set for method comparison. The remaining 224 sample pairs covering the concentration range $2.34-35.77 \mathrm{ng} / \mathrm{L}$ in plasma were used for method comparison.

The Passing-Bablok regression equation between whole blood and plasma hs-cTnI on the PATHFAST analyser showed a linear correlation (Figure 1) described by the following equation:

$$
\begin{gathered}
y=0.218+0.824 x \\
(y=\text { hs-cTnI plasma, } x=\text { hs-cTnI whole blood }) .
\end{gathered}
$$

Overall, the comparison between whole blood and plasma for hs-cTnI yielded a correlation coefficient ( $r$ ) of 0.99 (Figure 2). The bias measured for whole blood vs. plasma for hs-cTnI, were between +52 and $-82 \%$, respectively (Figure 3).

In the Bland Altman plot (Figure 3), the limits of agreement (LoA) did not exceed the maximum allowed difference of $90 \%$ between methods $(\Delta)$. As such, results from plasma and whole blood were considered to be in agreement, indicating that they can be used interchangeably. There was no systematic bias toward one method across the tested concentration range. 


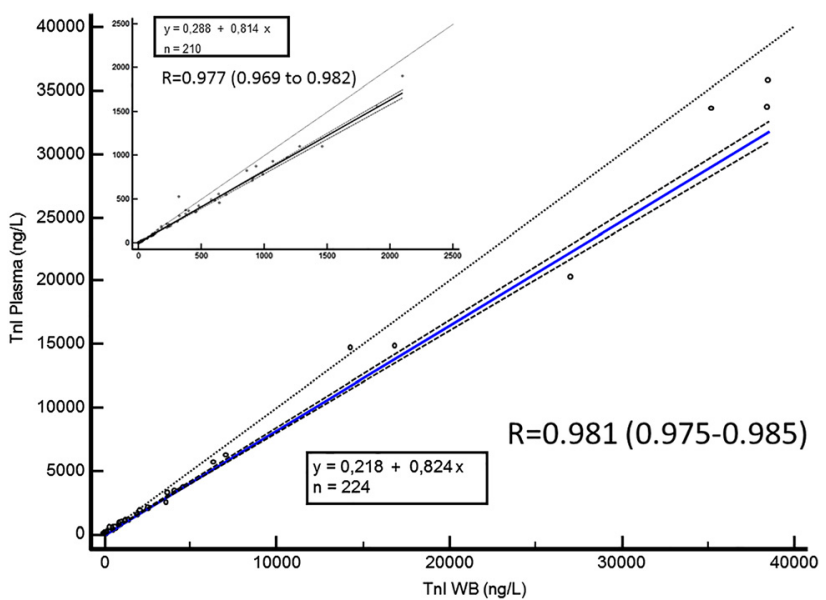

Figure 1: Passing-Bablok regression.

Calculation was executed on 224 paired plasma and whole blood hscTnl samples. The intercept is 0.218 and the slope is 0.824 . The dotted line denotes the line of equality. The blue line denotes the regression line with corresponding $95 \%$ confidence intervals. A second Passing-Bablok regression showing the regression of samples within the concentration range of $0-2,500 \mathrm{ng} / \mathrm{L}$ was added as an insert. The intercept of this regression is 0.288 and the slope is 0.814 . The correlation coefficient is $\mathrm{r}=0.977$ ( $95 \% \mathrm{Cl}: 0.969-0.982)$.

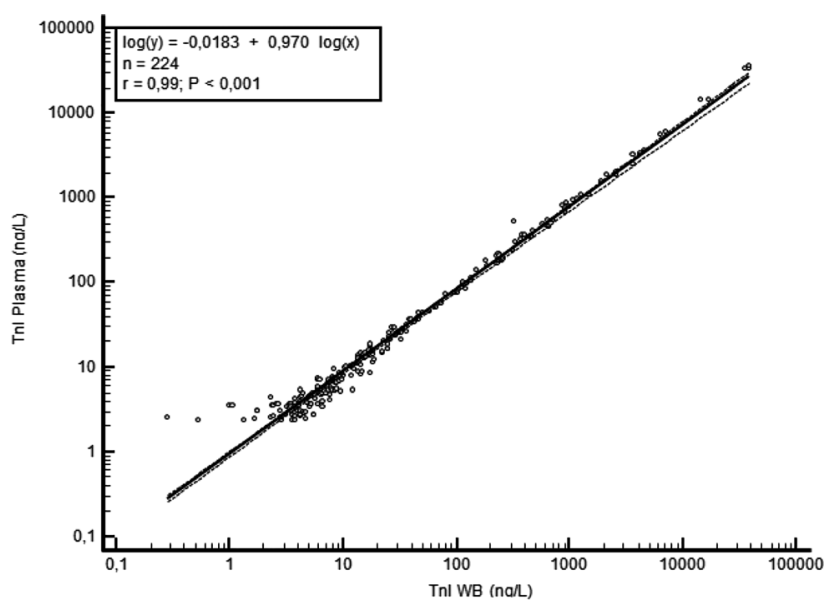

Figure 2: Spearman rank correlation with corresponding $95 \%$ confidence intervals between log transformed hs-cTnl in plasma and whole blood.

Inter-rater agreement between elevated and normal plasma and whole blood hs-cTnI based on the general 99th percentile URL $(24.2 \mathrm{ng} / \mathrm{L})$ yielded a weighted Cohen's Kappa of 0.93 (95\% CI: 0.89-0.98) which indicates a very good agreement $(>0.90)$ between methods (see Table 1$)$.

Precision evaluation of whole blood and plasma according to CLSI ep 15 was performed by serial measurements in parallel in whole blood and plasma on four different days with different cTnI concentrations by using suitable selected patient samples. Whole blood CVs were

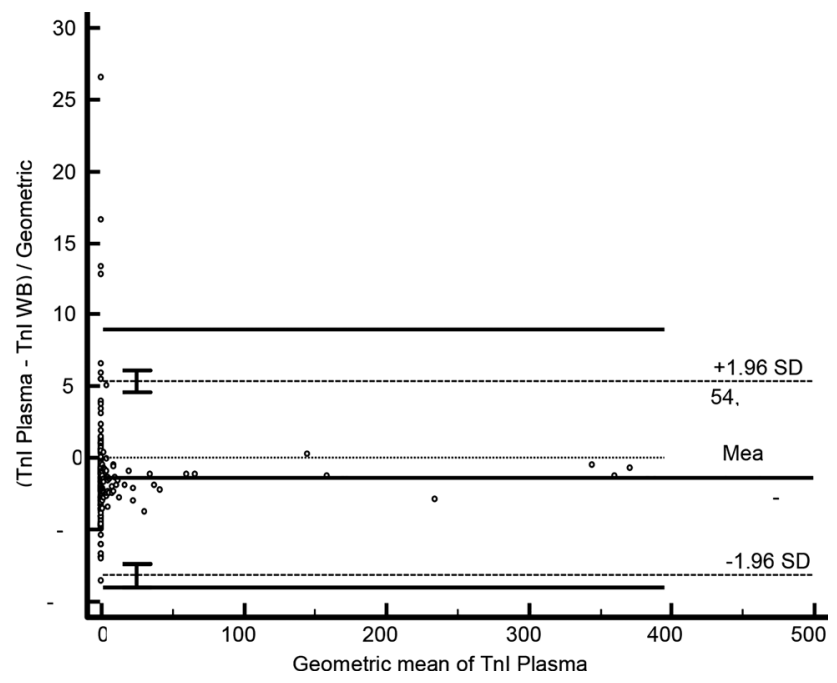

Figure 3: Bland Altman diagram.

The dotted lines denote the limit of agreement within 1.96 standard deviation and corresponding $95 \%$ confidence intervals. The straight line denotes the maximum allowed difference $(90 \%)$ between methods $(\Delta)$. The limits of agreement (LoA) did not exceed the maximum allowed difference between methods.

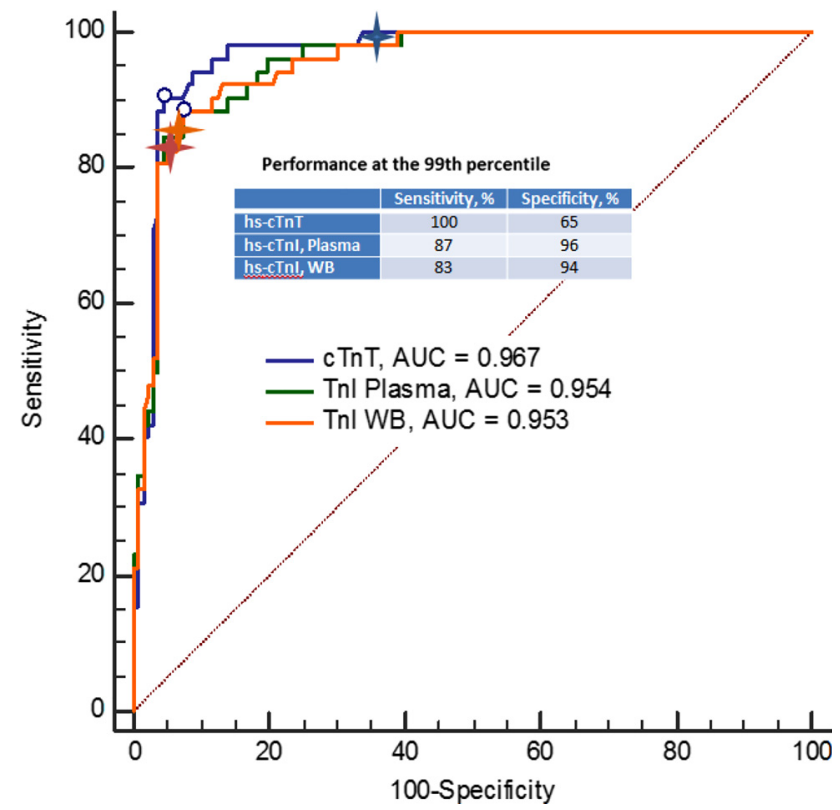

Figure 4: Receiver operating curve characteristics regarding performance of baseline hs-cTn measured with hs-cTnT, plasma hscTnl and whole blood hs-cTnl.

The location of the respective 99th percentile values is marked on the ROC curve.

17.7 and 5.2\% between mean concentrations of 3.8 and $16.4 \mathrm{ng} / \mathrm{L}$ (Figure 4). Of particular note is the finding that higher precision was measured in whole blood than in plasma. 


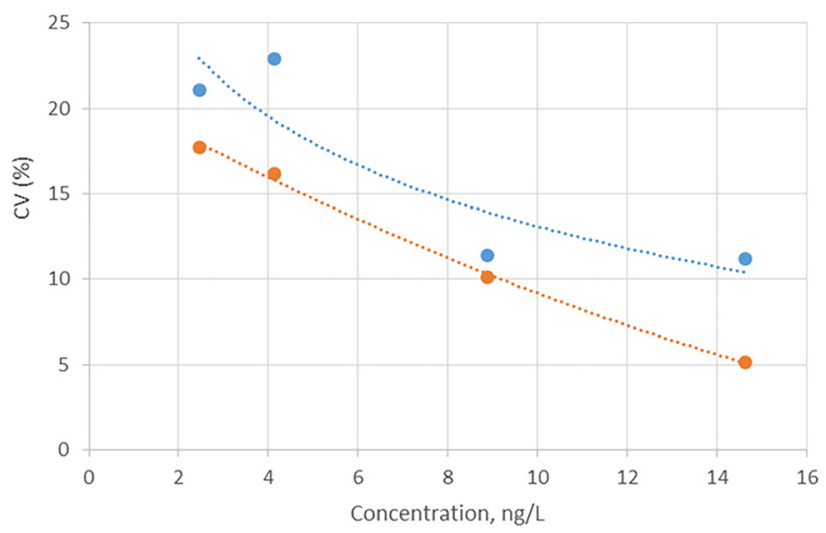

Figure 5: Imprecision profile of plasma and whole blood hs-cTnl in parallel by serial measurement according to CLSI ep 15 in four different patient samples on four different days (blue: plasma; yellow: whole blood).

\section{Discriminatory ability of hs-cTnl vs. hs-cTnT at presentation for discrimination of NSTEMI}

The diagnostic accuracy of measurements obtained at presentation, quantified by AUC was 0.954 (95\% CI: 0.914-0.979) for plasma hs-cTnI, 0.953 (95\% CI: 0.913-0.979) for whole blood, and 0.967 (95\% CI: 0.930-0.987) for hs-cTnT (Figure 3, Figure 5). Pairwise comparison of ROC curves showed no significant difference between areas $(\triangle \mathrm{AUC} 0.0009, \mathrm{p}=0.7$ for whole blood vs plasma hs-cTnI, $\triangle$ AUC 0.013 for plasma hscTnI vs hs-cTnT, $\mathrm{p}=0.3, \triangle \mathrm{AUC} 0.013, \mathrm{p}=0.3$ for whole blood hscTnI vs hs-cTnT).

\section{Discussion}

Until recently, POC-cTn assays had inferior analytical and clinical performance than central laboratory tests, mostly due to the lack of appropriate sensitivity for early and accurate diagnosis of MI. With the development of hs-cTn assays on POC technologies that show comparable analytical and clinical performance, POCT may emerge as an attractive option in hospitals that have no or only limited access to central laboratory measurements, or do not meet required turn-around-times between blood draw and reporting $[1,4,15]$. For convenience and in order to avoid the time-consuming preparation of plasma, the ability of a POCT analyser to process whole blood samples gains importance. Unfortunately, only sparse data are available on the performance of whole blood vs. plasma on POCT.

Therefore, our findings are important and corroborate the clinical usefulness of the PATHFAST hs-cTnI assay.
We report several important findings. First, the correlation between whole blood and plasma hs-cTnI is almost linear across the entire range of measurable concentrations. Second, although a bias between sample types exists, this bias did not exceed the maximum allowed difference between methods, and there was no systematic bias between plasma and whole blood suggesting that plasma and whole blood can be used interchangeably across a wide spectrum of hs-cTnI concentrations. Third, the inter-rater agreement between whole blood and plasma above or below the 99th percentile URL yields a weighted Cohen's Kappa of 0.93 which has to be interpreted as very good.

Fourth, we compared the diagnostic performance of the initial blood sample on admission of hs-cTnI in plasma and whole blood with hs-cTnT to discriminate a final diagnosis of NSTEMI based on serial hs-cTnT. The ROC analysis showed excellent performance of all three tests with AUC between 0.953 and 0.967, without a significant difference. This finding confirms and corroborates recent findings on the excellent clinical performance for the diagnosis of MI by the use of PATHFAST hs-cTnI as POCT [5, $6,16]$. The PATHFAST hs-cTnI has a validated ESC $0 / 1 \mathrm{~h}$ protocol for the diagnosis of NSTEMI [5] and has been listed among the available hs-cTnI assays that are being recommended by 2020 ESC Guidelines on NSTE-ACS for fast ruleout and rule-in of NSTEMI [1].

\section{Limitations}

The sample size of samples with high cTnI concentrations is low, and the reported results on agreement have to be verified for the high concentration range. However, assay imprecision of hs-cTn assays is higher in the low concentration range and it is therefore clinically more important to have congruent values close to the decision limit, i.e. the 99th percentile upper limit of normal.

The high areas under ROC curves indicating an excellent discrimination of NSTEMI depend on the selection of the study population and the pre-test probability for NSTEMI. A selection bias towards selection of patients with higher prevalence of a final NSTEMI diagnosis cannot be fully excluded. Therefore forthcoming studies are required to validate the findings of this pilot study in larger unselected populations presenting with chest pain.

The findings of this study regarding the performance of whole blood vs. plasma are assay specific for the Pathfast hs-cTnI assay and cannot be generalized to other hs-cTnI assays on POC platforms. 


\section{Conclusions}

With the Pathfast hs-cTnI assay, whole blood can be used interchangeably with plasma for more convenient and less time and labor-consuming testing of hs-cTnI in the ED. Method comparison suggests an excellent correlation between sample types with a small degree of underestimation of hs-cTnI concentration when measured by whole blood. However, this underestimation and a considerable bias do not affect agreement of results above or below the relevant 99th percentile ULN. In addition, the clinical performance of whole blood and plasma hs-cTnI is excellent without a difference for discrimination of NSTEMI.

Acknowledgments: We thank the study nurses Heidi Deigentasch, Elisabeth Mertz and Amelie Werner for assistance to conduct the laboratory experiments.

Research funding: None declared.

Author contributions: All authors have accepted responsibility for the entire content of this manuscript and approved its submission.

Competing interests: EG declares honoraria for lectures from Daiichi Sankyo, Astra Zeneca, Roche Diagnostics, Boehringer Ingelheim, Bayer Vital, and BRAHMS GmbH. He receives research funding from Daiichi Sankyo and Roche Diagnostics. He consults Roche Diagnostics, Astra Zeneca, Bayer Vital, Indorsia, Radiometer, BRAHMS GmbH, Hoffmann-La Roche, and Boehringer Ingelheim. NF received lecture honoraria from AstraZeneca, BayerVital, Boehringer Ingelheim and Novartis. ES receives research funding from Axis Shield Diagnostics, Mitsubishi Chemical Europe, Radiometer, Roche Diagnostics, Saladax Biomedical, Shanghai Kehua Bio-engineering. All other authors state no conflict of interest.

Informed consent: Informed consent was obtained from all individuals included in this study.

Ethical approval: The local Institutional Review Board has given permission to conduct this study.

\section{References}

1. Collet JP, Thiele H, Barbato E, Barthélémy O, Bauersachs J, Bhatt DL, et al. ESC Guidelines for the management of acute coronary syndromes in patients presenting without persistent ST-segment elevation. Eur Heart J 2020 Aug 29:ehaa575. https://doi.org/10. 1093/eurheartj/ehaa575 [Epub ahead of print. PMID: 32860058].

2. Stoyanov KM, Biener M, Hund $H$, Mueller-Hennessen M, Vafaie $M$, Katus HA, et al. Effects of crowding in the emergency department on the diagnosis and management of suspected acute coronary syndrome using rapid algorithms: an observational study. BMJ Open 2020;10:e041757.

3. Wu AHB, Christenson RH, Greene DN, Jaffe AS, Kavsak PA, Ordonez-Llanos J, et al. Clinical laboratory practice recommendations for the use of cardiac troponin in acute coronary syndrome: expert opinion from the Academy of the American Association for Clinical Chemistry and the task force on clinical applications of cardiac bio-markers of the International Federation of Clinical Chemistry and Laboratory Medicine. Clin Chem 2018;64:645-55.

4. Collinson PO, Saenger AK, Apple FS, IFCC C-CB. High sensitivity, contemporary and point-of-care cardiac troponin assays: educational aids developed by the IFCC Committee on Clinical Application of Cardiac Bio-Markers. Clin Chem Lab Med 2019;57: 623-32.

5. Sörensen NA, Neumann JT, Ojeda F, Giannitsis E, Spanuth E, Blankenberg S, et al. Diagnostic evaluation of a high-sensitivity troponin I point-of-care assay. Clin Chem 2019;65:1592-601.

6. Boeddinghaus J, Nestelberger T, Koechlin L, Wussler D, LopezAyala P, Walter JE, et al. Early diagnosis of myocardial infarction with point-of-care high-sensitivity cardiac troponin I. J Am Coll Cardiol 2020;75:1111-24.

7. Venge $P$, van Lippen L, Blaschke S, Christ M, Geier F, Giannitsis E, et al. Equal clinical performance of a novel point-of-care cardiac troponin I (cTnl) assay with a commonly used high-sensitivity cTnI assay. Clin Chim Acta 2017;469:119-25.

8. https://www.accessdata.fda.gov/cdrh_docs/reviews/K100130. pdf [Accessed 16 Feb 2020].

9. Christenson RH, Mullins K, Duh SH. Validation of high-sensitivity performance for a United States Food and Drug Administration cleared cardiac troponin I assay. Clin Biochem 2018;56:4-10.

10. Thygesen K, Alpert JS, Jaffe AS, Chaitman BR, Bax JJ, Morrow DA, et al. Fourth universal definition of myocardial infarction (2018). Eur Heart J 2019;40:237-69.

11. Saenger AK, Beyrau R, Braun S, Cooray R, Dolci A, Freidank H, et al. Multicenter analytical evaluation of a high-sensitivity troponin T assay. Clin Chim Acta 2011;412:748-54.

12. http://labogids.sintmaria.be/sites/default/files/files/troponin_ t_hs_2019-02_v8.pdf [Accessed 16 Feb 2020].

13. Giannitsis E, Mueller-Hennessen M, Zeller T, Schuebler A, Aurich $M$, Biener $M$, et al. Gender-specific reference values for highsensitivity cardiac troponin T and I in well-phenotyped healthy individuals and validity of high-sensitivity assay designation. Clin Biochem 2020;78:18-24.

14. Bablok W, Passing H, Bender R, Schneider B. A general regression procedure for method transformation. Application of linear regression procedures for method comparison studies in clinical chemistry, part III. J Clin Chem Clin Biochem 1988;26:783-90.

15. Apple FS, Fantz CR, Collinson PO. IFCC Committee on Clinical Application of Cardiac Bio-Markers. Implementation of highsensitivity and point-of-care cardiac troponin assays into practice: some different thoughts. Clin Chem 2020 Dec 5: hvaa264. https://doi.org/10.1093/clinchem/hvaa264 [Epub ahead of print. PMID: 33279984].

16. Pickering JW, Young JM, George PM, Watson AS, Aldous SJ, Troughton RW, et al. Validity of a novel point-of-care troponin assay for single-test rule-out of acute myocardial infarction. JAMA Cardiol 2018;3:1108-12. 